\title{
Impedanzspektroskopie eines auf metallorganischen Netzwerken basierenden Sorptionssensors
}

\author{
H. Lensch ${ }^{1}$, N. Baskent ${ }^{2}$, I. Wilhelm ${ }^{3}$, J. Huerttlen ${ }^{3}$, M. Voelker $^{2}$, A. Schütze ${ }^{1}$, T. Sauerwald ${ }^{1}$ \\ ${ }^{1}$ Lehrstuhl für Messtechnik, Campus A5.1, Universität des Saarlandes, 66123 Saarbrücken \\ E-Mail-Adresse, h.lensch@lmt.uni-saarland.de \\ ${ }^{2}$ Fraunhofer-Institut für Integrierte Schaltungen (IIS) Am Wolfsmantel 3391058 Erlangen \\ ${ }^{3}$ Fraunhofer-Institut für Chemische Technologien (ICT) Joseph-von-Fraunhofer-Str. 776327 Pfinztal
}

\section{Zusammenfassung}

Es wird ein auf metallorganischen Netzwerken (MOF) basierender Sensor präsentiert, auf welchem flüchtige organische Verbindungen (VOC) aufgrund von hohen Verteilungskoeffizienten sehr gut sorbieren können. Der Sensor besteht aus einem keramischen Substrat, auf dem Interdigital-Elektroden aufgebracht sind. Auf diesen Elektroden wird das mit Bindern versetzte MOF UIO-66 mittels Airbrush Verfahren aufgesprüht. Die Kapazität des Sensors im Bereich von 14,9 pF bis 15,4 pF bei einem Ethanolangebot von $0 \mathrm{ppm}$ bis $1.000 \mathrm{ppm}$ wird mittels Impedanzspektroskopie gemessen. Weiterhin wird die Querempfindlichkeit auf Feuchte $(40 \% \mathrm{rH}$ und $60 \% \mathrm{rH})$ untersucht, welche zusätzlich zu einer starken Änderung der Kapazität (auf bis zu 24,3 pF) auch noch einen zusätzlichen resistiven Signalanteil aufweist. Mit Hilfe von Impedanzspektroskopie, können daher die kapazitiven und resistiven Beiträge der Feuchte separat bestimmt werden. Dies eröffnet die Möglichkeiten, um den Einfluss der Feuchte zu kompensieren.

Keywords: Gassensoren, VOC-Messung, Metal-organic frameworks, Impedanzspektroskopie

\section{Einleitung}

Das Messen von flüchtigen organischen Verbindungen (engl. Volatile organic compounds - VOCs) gewinnt aufgrund von der von ihnen ausgehenden Gesundheitsrisiken zunehmend an Bedeutung. Eine Herausforderung beim Messen dieser Stoffe ist die niedrige Konzentration der Einzelkomponenten und die große Vielfalt an möglichen Zielgasen [1], [2]. Metallorganische Netzwerke (engl. Metal-Organic Framework MOF) eignen sich ausgesprochen gut zum sorbieren von VOCs, da die erzielten Verteilungskoeffizienten um Größenordnungen oberhalb denen klassischer Sorptionsmaterialien liegen können [3]. Darüber hinaus stellen MOFs sterisch und chemisch auf bestimmte VOC zugeschnittene Adsorptionsplätze bereit [4]. Dies ermöglicht ein neues Sensorkonzept für VOCs basierend auf einer direkten Sorptionsmessung.

Die kapazitive Auslesung von Sorptionssensoren für Feuchte ist gut beschrieben, da diese eine hohe Änderung der Permittivität verursacht [5]. Neben diesem kapazitiven Signalanteil führt die Feuchtesorption auch zu einer Änderung der ionischen Leitfähigkeit [6], was für VOC-Sensoren zur Kompensation der
Feuchtequerempfindlichkeit genutzt werden kann, die eine Messung sonst erheblich stört. In dieser Arbeit wird eine erste Charakterisierung eines MOF-basierten Sorptionssensor mittels Impedanzspektroskopie vorgestellt und im Rahmen eines vereinfachten Leitungsmodells interpretiert, das als Basis für eine Kompensation der Querempfindlichkeit auf Feuchte dienen kann.

\section{Sensordesign}

Der Sorptionssensor weist drei InterdigitalElektroden (Ana, Ref, Com) auf, welche nebeneinander auf einem Substrat aufgebracht sind. Dabei bildet jeweils eine Elektrode zusammen mit der gemeinsamen Elektrode (COM) einen Kondensator (Abbildung 1). Eines der Elektrodenpaare (Ana - Com) wird mit einer sensitiven Schicht versehen und das andere Paar bleibt unbeschichtet (Com - Ref), um für eine spätere differentielle Messanordnung zur Verfügung zu stellen. Als Substrat wird eine keramische Leiterplatte verwendet (Rogers4350B, rel. Permittivität 3.48 0.05 @ $10 \mathrm{GHz})$. 

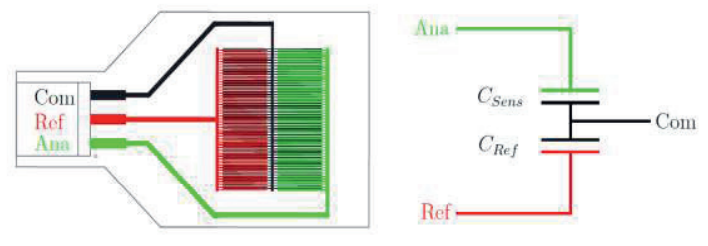

Abbildung 1: Sensoraufbau (links) mit elektrischen Ersatzschaltbild der Anordnung (rechts).

Als sensitive Schicht wurde die mikroporöse metall-organische Gerüstverbindung (MOF) UiO-66 verwendet, welche mit Bindern zu einer viskosen Paste verarbeitet und via AirbrushVerfahren auf Interdigital-Elektroden aufgetragen wurde. Durch die Anzahl der Beschichtungsvorgänge lässt sich die Dicke des sensitiven Materials einstellen, wobei pro pro Beschichtung ca. $1 \mu \mathrm{m}$ aufgetragen wird. Für erste Untersuchungen wurde eine Schichtdicken von ca. $2 \mu \mathrm{m}$ bis $20 \mu \mathrm{m}$ eingestellt, die auf die Elektroden mit einer Metalldicke von $35 \mu \mathrm{m}$ sowie einer Breite und einem Abstand von $75 \mu \mathrm{m}$ aufgebracht wurden.

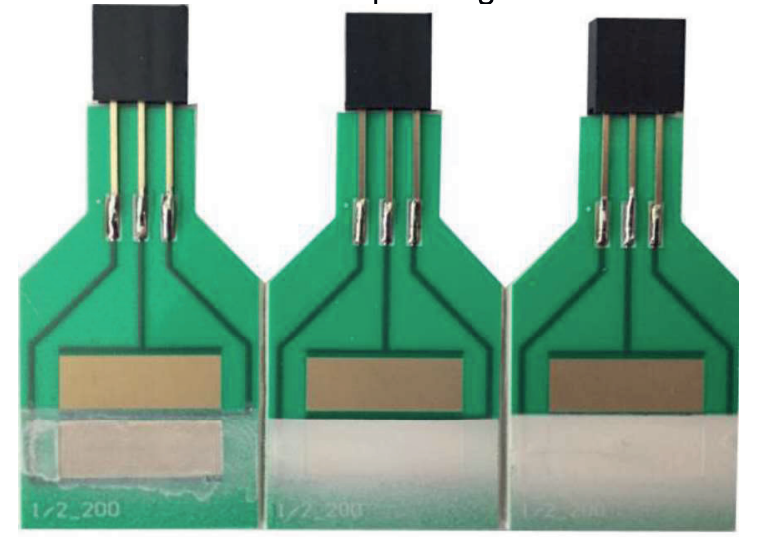

Abbildung 2: Sensor mit 2 (links), 10 (Mitte) und 20 (rechts) MOF-Schichten

\section{Experimenteller Aufbau}

Um den Sensor mit verschiedenen Testgasen $\mathrm{zu}$ beaufschlagen wurde dieser in eine luftdichte Messkammer aus Aluminium eingebaut und mit Hilfe einer Flachdichtung aus Viton auf der Platine gedichtet, so dass die elektrischen Anschlussbuchsen herausragen. An die Pins Ana und Com wurde direkt das Agilent 4294A bzw. Solartron 1260 Impedanzspektrometer angeschlossen.

Die Gasanschlüsse der Messkammer wurden mit einer Gasmischanlage verbunden, mit der eine Gasmischung aus verschiedenen Testgasen bei verschiedenen relativen Feuchten angeboten werden kann.

\section{Modell}

Durch Sorption eines Gases an der Gerüstverbindung ändert sich die Permittivität $\varepsilon_{r}$ der Sensorschicht.

Diese Änderung führt zu einer messbaren Erhöhung der Kapazität [7]. Für nichtleitende Adsorbate ändert sich nur der Realteil der Permittivität und der Sensor verhält sich im gesamten Impedanzspektrum als idealer Kondensator. Dies entspricht im BodeDiagramm einer Steigung von -1 in der Amplitude und mit einem Phasenwinkel von $-90^{\circ}$.

Die Adsorption von Feuchte führt zu einer ausgesprochen hohen Änderung der Permittivität bzw. Erhöhung der Kapazität, da Feuchte sehr polar ist, ein Umstand der auch in kapazitiven Feuchtesensoren genutzt wird. Trotz der Verwendung von Materialien, die unpolare VOC bevorzugt adsorbieren, führt ein rein kapazitives Auslesen der Sensoren zu einer sehr ausgeprägten Querempfindlichkeit auf Feuchte. Zur selektiven Bestimmung zwischen Gas und Feuchte muss also zumindest ein weiterer Sensorparameter verwendet werden. In [6] wurde gezeigt, dass adsorbierte Feuchte auf $\mathrm{TiO}_{2}$ zusätzlich zu dem kapazitiven Verhalten noch weitere Signalanteile besitzt, u.a. ein sogenanntes Constant Phase Element, welches eine Überlagerung von rein realen und rein imaginären frequenzabhängigen Impedanzen mit konstantem Phasenwinkel ergibt. Dieser Signalanteil wird mit der ionischen Leitfähigkeit der Wassermoleküle auf der Oberfläche gedeutet (Grothuss Mechanismus). Da es sich hierbei also um einen Effekt vom physisorbiertem Wasser handelt, erwarten wir, dass dieser Signalanteil bei einer Vielzahl von Materialien, z.B. den hier verwendeten MOFs auftritt. Mittels Fit an ein passendes Ersatzschaltbild können CPE und Kapazität aus der Gesamtimpedanz getrennt werden, da die ionischen Effekte in niedrigeren Frequenzbereichen dominanter sind als der für VOC Detektion interessante kapazitive Anteil [7],[8]. So entstehen weitere Merkmale im Spektrum, die selektiv auf Feuchte sind. Diese können genutzt werden, um den Feuchteanteil der Impedanz im rein kapazitiven Bereich zu kompensieren.

\section{Ergebnisse}

Alle Messungen wurden mit dem Sensor mit 20 Schichten durchgeführt, da anzunehmen ist, dass dort, durch die größte Zahl an Adsorptionsplätzen die Änderung der Kapazität am größten ist. Alle Messungen wurden bei Raumtemperatur durchgeführt. 
Abbildung 3 zeigt ein Bode Diagramme des Sensors aufgenommen mit dem Agilent 4294A Impedanzspektrometer im Frequenzbereich von $40 \mathrm{~Hz}$ bis $125 \mathrm{MHz}$. Die Messung wird in Stickstoff mit Reinheit 5.0. Die Amplitude hat bei allen aufgenommenen Spektren eine Steigung von -1 und einen Phasenwinkel von $-90^{\circ}$, was auf ein rein kapazitives Verhalten hinweist. Unter der Annahme eines idealen Kondensators wurde anschließend die Kapazitäten des Sensors bestimmt (Tabelle 1).
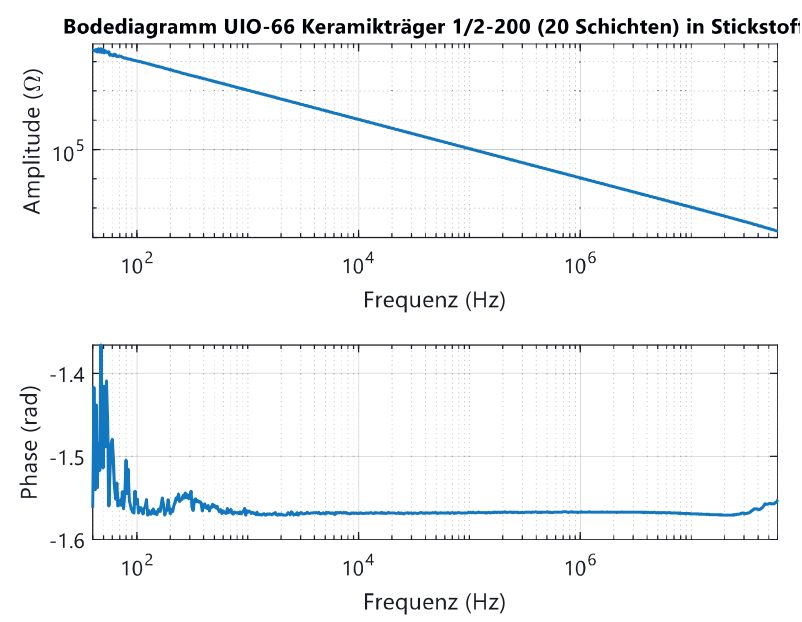

\section{Abbildung 3: Sensor mit Schicht in Stickstoff 5.0}

Um das zeitliche Verhalten zu charakterisieren wurde eine Messung in Stickstoff 5.0 und verschiedenen Ethanolkonzentrationen $(0 \mathrm{ppm}$, 200 ppm, 500 ppm, 1000 ppm) durchgeführt. Die Konzentration wurden jeweils nach 30 min erhöht. Die Spektren wurden mit dem Agilent 4294A Impedanzspektrometer aufgenommen, da dieses pro Spektrum weniger Zeit als das Solartron benötigt und so das dynamische Verhalten besserabgebildet werden kann. Anschließend wurde aus den Spektren die Kapazität bestimmt und für $520 \mathrm{kHz}$ den zeitlichen Verlauf der Kapazität aufgetragen (Abbildung 4).

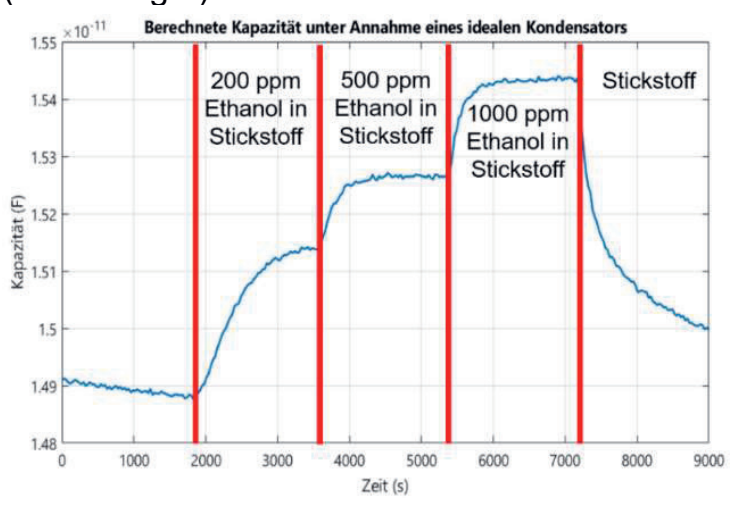

Abbildung 4: Zeitlicher Verlauf der Kapazität des Sensors mit MOF-Schicht bei unterschiedlichen Ethanolangeboten bei $520 \mathrm{kHz}$
Nachdem der Sensor bei trockener Umgebung charakterisiert wurde, wird im Folgenden der Einfluss auf Feuchte untersucht. Um Messung mit einer niedrigeren Grenzfrequenz $(1 \mathrm{~Hz}) \mathrm{zu}$ ermöglichen wurden die Messungen mit einem Solatron 1260 durchgeführt. In Abbildung 5 sind zwei Spektren bei jeweils $40 \% \mathrm{rH}$ und $60 \% \mathrm{rH}$ abgebildet. Diese zeigen im oberen Frequenzbereich $(a b 4 \mathrm{kHz})$ ein rein kapazitives Verhalten. Im mittleren Bereich $(5 \mathrm{~Hz}$ bis $200 \mathrm{~Hz}$ ) bildet sich bei $60 \% \mathrm{rH}$ ein deutlich resistiver Bereich aus und im unteren Bereich wird die Impedanz leicht kapazitiver.

Bei $40 \% \mathrm{rH}$ wird der mittlere resistive Bereich erst nahe der unteren Grenzfrequenz ausgebildet, was an dem Abflachen der Amplitude und dem Rückgang der Phase Richtung $0^{\circ}$ erkennbar ist. Für detailliertere Untersuchungen ist es allerdings notwendig den untersuchten Frequenzbereich weiter nach unten zu erweitern.

Tabelle 1 Kapazitäten bei verschiedenen Gasangeboten

\begin{tabular}{|l|r|}
\hline Sensor & $\begin{array}{r}\text { Kapazität } \\
\text { [nF] }\end{array}$ \\
\hline Trocken in Stickstoff & 14.9 \\
\hline $200 \mathrm{ppm}$ Ethanol in Stickstoff & 15.11 \\
\hline $500 \mathrm{ppm}$ Ethanol in Stickstoff & 15.25 \\
\hline $1000 \mathrm{ppm}$ Ethanol in Stickstoff & 15.4 \\
\hline $40 \% \mathrm{rH}$ in Stickstoff & 20.71 \\
\hline $60 \% \mathrm{rH}$ in Stickstoff & 24.30 \\
\hline
\end{tabular}

\section{Diskussion \& Fazit}

Die Messungen in Abbildung 3 und Abbildung 4 zeigen ein rein kapazitives Verhalten des Sensors bei verschiedenen Konzentrationen von Ethanol. Obwohl sich der Sensor nicht linear verhält, ist eine klare Quantifizierung der Konzentration möglich. Die Empfindlichkeit von $15 \mathrm{pF}$ zwischen 0 und $1000 \mathrm{ppm}$ Ethanol ist zwar ausreichend, allerdings sind die hier untersuchten Konzentrationen relativ hoch und liegen z.B. oberhalb derer, die in eine Messung der Innenluft potenziellen Anwendung relevant sind. Es kann außerdem aufgrund der zu wählenden Gitterstruktur des MOF keine Unterscheidung von Gasen mit ähnlicher oder kleinerer Molekülgröße erfolgen. Aus diesem Grund zeigen MOFs ohne weitere Funktionalisierung eine geringe Selektivität. Auch optisch ausgelesene Sorptionssensoren wie in [9] beschrieben zeigen für leichtflüchtige VOC eine niedrigere Nachweisschwelle als alternative Sensoren, wie Halbleitergassensor oder Photoionisationsdetektor. Allerdings nimmt die Nachweisempfindlichkeit mit sinkender Flüchtigkeit sehr stark zu. Wir erwarten, dass kapazitiv ausgelesene 


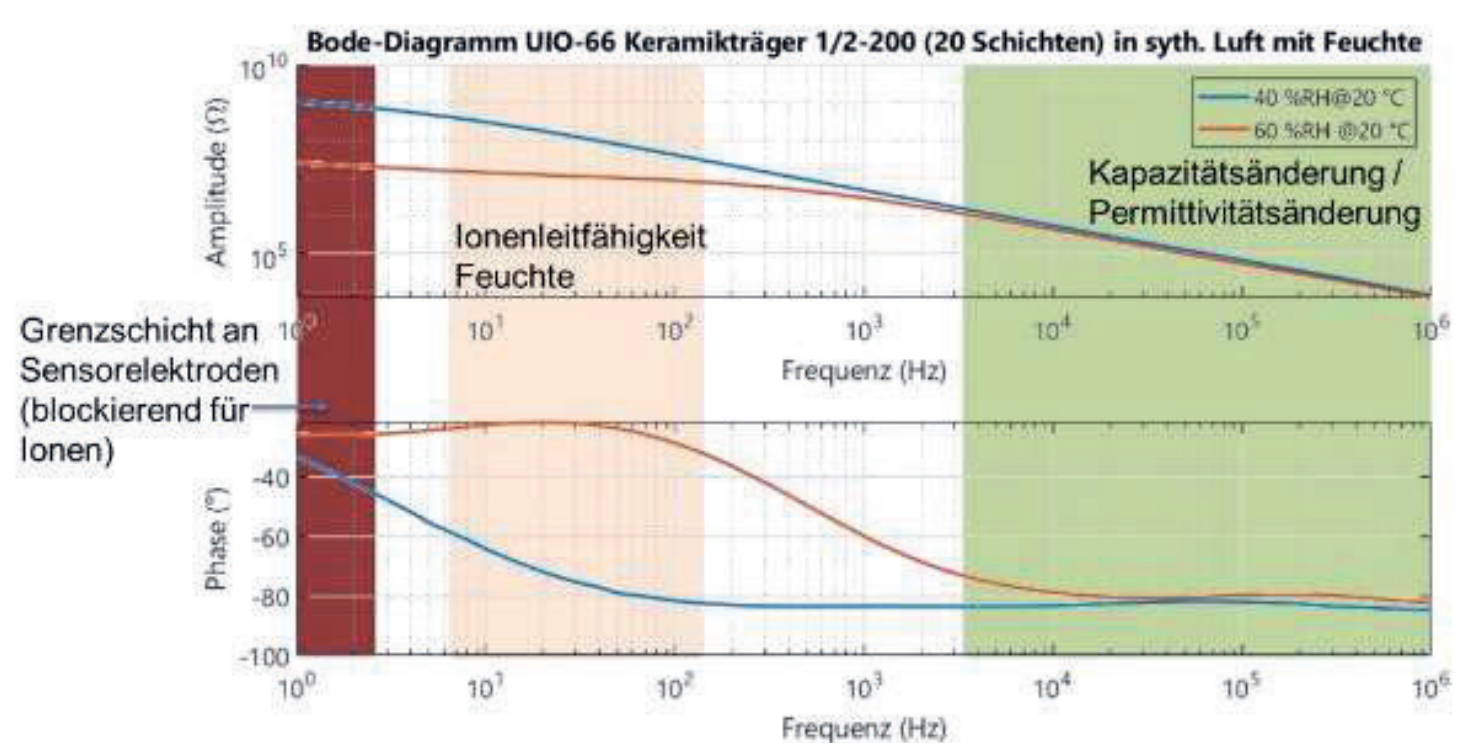

Abbildung 5: Bode Diagramm der Sensorschicht bei $40 \%$ rH (blau) und $60 \%$ rH (rot)

Sensoren v.a. für schwerflüchtige $\mathrm{VOC}$ eine Ergänzung zu bestehenden Konzepten sind, da für diese Stoffgruppen sehr hohe Verteilkoeffizienten zu erwarten sind. Für VOC in niedrigen Konzentrationen können auch kombinierte System verwendet werden [3]. In denen der MOF als Präkonzentrator benutzt wird und ein hochempfindlicher Halbleitergassensor als Detektor verwendet wird. In einem solchen System kann eine Messung des Präkonzentrators mit Impedanzspektroskopie zusätzliche Informationen über die Menge an sorbierten Stoffen liefern. Der Einfluss von Feuchte auf den Sensor ist vergleichbar mit dem in [6] beschriebenen Feuchtesensoren, obwohl es sich um ein völlig anderes Material handelt. Dies stützt die Vermutung, dass der Sensormechanismus in beiden Fällen auf eine Leitfähigkeit von physisorbiertem Wasser zurückzuführen ist. In Abbildung 5 hat der Feuchteeffekt soweit im Frequenzbereich abgebildet dem Modell aus [6] ähnelt. Impedanzspektroskopie als Messprinzip zum Auslesen des Sensors funktioniert sowohl bei der Sorption von Ethanol als auch von Feuchte. Feuchte liefert zusätzliche Merkmale in anderen Frequenzbereichen als Ethanol im Spektrum. Die Sensitivität auf Feuchte ist in dem untersuchten Messbereich deutlich größer als die auf die Zielgas, was eine Kompensation erschwert.

\section{Danksagung}

Die Entwicklung und Fertigung der Sensoren erfolgte im Rahmen des Forschungsvorhabens "Technologien für das Internet der Sinne" gefördert durch das Bundesland Bayern.

\section{Literaturnachweis}

[1] J. A. Bernstein et al., "The health effects of nonindustrial indoor air pollution," J. Allergy Clin. Immunol., vol. 121, no. 3, pp. 585-591, 2008.

[2] A. Schütze et al., "Highly Sensitive and Selective VOC Sensor Systems Based on Semiconductor Gas Sensors: How to?," Environments, vol. 4, no. 1, p. 20, 2017.

[3] M. Leidinger, M. Rieger, T. Sauerwald, C. Alépée, and A. Schütze, "Integrated preconcentrator gas sensor microsystem for ppb level benzene detection," Sensors Actuators, B Chem., vol. 236, pp. 988-996, 2016.

[4] J. L. C. Rowsell and O. M. Yaghi, "Metalorganic frameworks: A new class of porous materials," Microporous and Mesoporous Materials, vol. 73, no. 1-2. pp. 3-14, 2004.

[5] T. Wagner et al., "A high temperature capacitive humidity sensor based on mesoporous silica," Sensors, vol. 11, no. 3, pp. 3135-3144, 2011.

[6] H. Lensch, B. Manuel, A. Schütze, and T. Sauerwald, "Impedance model for a high temperature ceramic humidity sensor," $J$. Sensors Sens. Syst., vol. 8, no. Special issue Sensors and Measurement Systems 2018, pp. 1-14, Apr. 2019.

[7] C. Sapsanis et al., "Insights on capacitive interdigitated electrodes coated with MOF thin films: Humidity and VOCs sensing as a case study," Sensors (Switzerland), vol. 15, no. 8, pp. 18153-18166, Jul. 2015.

[8] E. Barsoukov and J. R. Macdonald, Impedance Spectroscopy: Theory, Experiment, and Applications, 2nd ed. Wiley-VCH-Verlag, 2005.

[9] DIN Deutsches Institut für Normung e. V., "Indoor air - - Part 29: Test methods for VOC detectors," no. 40, Berlin: Beuth Verlag, 2013, p. 44. 